\title{
Posterior Direct Decompression and Fusion of the Lower Thoracic and Lumbar Fractures with Neurological Deficit
}

\author{
Deuk Soo Jun, Chang Hun Yu, Byoung Geun Ahn \\ Department of Orthopedic Surgery, Gil Medical Center, Gachon University of Medicine and Science, Incheon, Korea
}

Study Design: A retrospective study.

Purpose: To analyze the treatment outcome of patients with lower thoracic and lumbar fractures combined with neurological deficits.

Overview of Literature: Although various methods of the surgical treatment for lower thoracic and lumbar fractures are used, there has been no surgical treatment established as a superior option than others.

Methods: Between March 2001 and August 2009, this study enrolled 13 patients with lower thoracic and lumbar fractures who underwent spinal canal decompression by removing posteriorly displaced bony fragments via the posterior approach and who followed up for more than a year. We analyzed the difference between the preoperative and postoperative extents of canal encroachment, degrees of neurologic deficits and changes in the local kyphotic angle.

Results: The average age of the patients was 37 years. There were 10 patients with unstable burst factures and 3 patients with translational injuries. Canal encroachment improved from preoperative average of $84 \%$ to $9 \%$ postoperatively. Local kyphosis also improved from $20.5^{\circ}$ to $1.5^{\circ}$. In $92 \%(12 / 13)$ of the patients, neurologic deficit improved more than Frankel grade 1 and an average improvement of 1.7 grade was observed. Deterioration of neurologic symptoms was not observed. Although some loss of reduction of kyphotic deformity was observed at the final follow-up, serious complications were not observed.

Conclusions: When posteriorly displaced bony fragments were removed by the posterior approach, neurological recovery could be facilitated by adequate decompression without serious complications. The posterior direct decompression could be used as one of treatments for lower thoracic and lumbar fractures combined with neurologic injuries.

Key Words: Lower thoracic and lumbar, Unstable burst fracture, Posterior direct decompression, Neurologic manifestations

\section{Introduction}

There exist several methods for the treatment of lower thoracic and lumbar fractures. In general, conservative management is recommended for patients with stable and neurologically free fractures. However, for patients who experi- ence neurologic deficits, surgical intervention is mostly recommended. After deciding to operate on patients who sustained fractures combined with neurologic deficits, an appropriate surgical approach is needed to be decided. Although three approaches, namely anterior, posterior and combined approach exist, it is still controversial to say what can be an ideal method. It has been stated that the anterior

Received Sep 20, 2010; 1st Revised Nov 15, 2010; Accepted Nov 18, 2010

Corresponding author: Chang Hun Yu, MD

Department of Orthopaedic Surgery, Gil Medical Center, Gacheon University of Medicine and Science,

1198 Guwol-dong, Namdong-gu, Incheon 406-799, Korea

Tel: +82-32-460-8201, Fax: +82-32-468-5437, E-mail: changhun.yu@gmail.com

* Excerpts of this paper was presented at the 53rd Korean Orthopedic Association in 2009. 
approach is more useful for decompression of the neural structure [1,2]. However, it has also been claimed that there is no significnat difference in clinicial outcomes of the anterior appraoch and those of the posterior approach on grounds that sufficient decompression could be achieved via posterior approach with anterior impaction of displaced bony fragments [3]. However, when impaction of bony fragments is performed, it remains challenging to achieve adequate decompression in some patients, and post-operatively re-displacement of the impacted bony fragments may occur.

The possibility of recovery from complete cord injury remains low. As such, it has been proposed that for patients who sustain complete spinal cord injuries, the focus of treatment should be on the vertebral body alignment and stability, rather than neural decompression. It has also been reported that even in patients with complete cord injuries, better neurological recovery could be achieved when sufficient decompression was performed [4]. This study examined the degrees of neurological recovery and radiological parameters indicating, facture union and correction of kyphotic deformity in patients with complete or incomplete neurologic deficits triggered by lower thoracic and lumbar fractures who underwent removal of posteriorly displaced

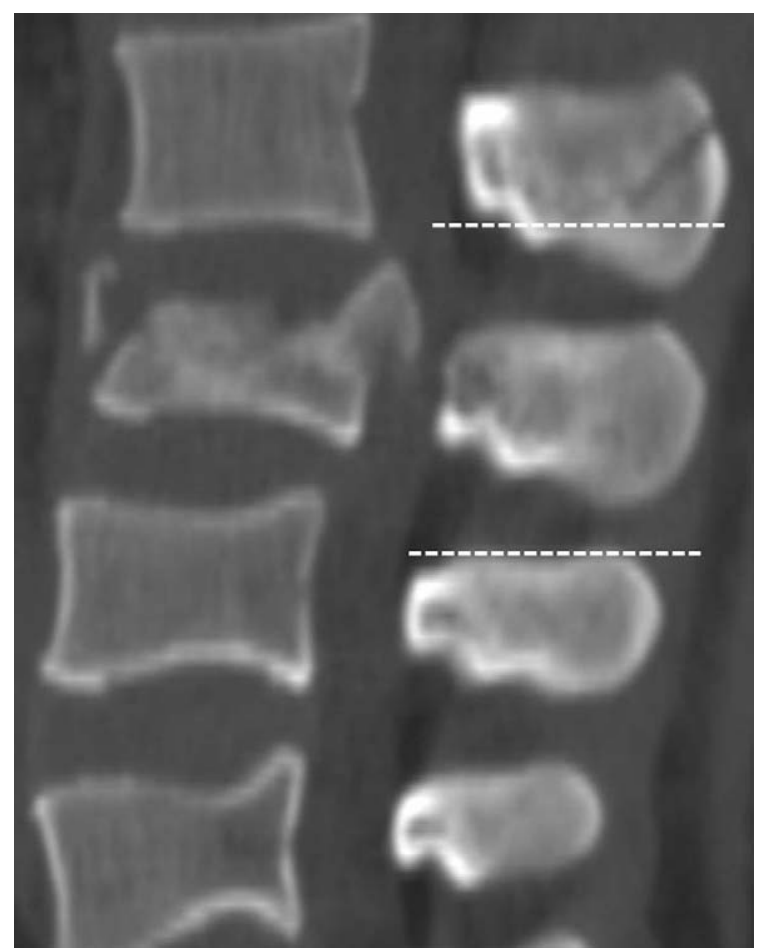

Fig. 1. Preoperative planning of laminectomy. Total laminectomy is performed on the fratured vertebra and partial laminectomy on the upper level. bony fragment, not impaction for spinal canal decompression.

\section{Materials and Methods}

\section{Study subjects}

We analyzed 232 patients admitted to our hospital with lower thoracic and lumber fractures and underwent surgery between March 2001 and August 2009. Among these patients, simple posterior instrumentation and fusion were performed in 199 patients (86\%). Thirty three patients (14\%) with complete or incomplete neurologic deficits were subject to the posterior approach for decompression, instrumentation and fusion. Among them, 15 patients underwent sole laminectomy. Laminectomy and direct decompression was performed in the rest 18 patients. Among the 18 patients who underwent direct spinal cord decompression, 13 patients were available for follow-up visits for more than one year. All operations were performed by the first author.

\section{Surgical methods}

The conventional posterior approach using midline incision was applied. For patients whose anterior vertebral comminution was not severe, two-level fusion was performed. For patients with thoracolumbar junction or severe vertebral body comminution, three- or four-level fusion was performed. Pedicle screws were inserted into the superior and inferior adjacent vertebrae. Total laminectomy was performed on the fractured vertebrae and partial laminectomy was performed on the superior adjacent vertebrae (Fig. 1). Dural repair was performed on patients who sustained dura tear. Approach started on the side with apparently severe neurologic symptoms or huge bony fragments on pre-operative computed tomography (CT). Partial or total pediculectomy was performed to directly inspect posteriorly displaced bony fragments and to avoid excess dural retraction that may cause secondary injury during removal of bony fragments. For the pedicle on the opposite side, except patients with comminuted fractures in the pedicle, shortlength and small-diameter pedicle screws were inserted to prevent the rod from receiving excessive load at the fracture sites. After carefully retracting the dura to the medial side, bony fragments were removed with pituitary forceps. For patients whose bony fragments were too large, thus excessive retraction was required and additional nerve injury 
could occur, the size was reduced by using Kerrison punch or osteotome and then subsequently removed. After adequate decompression, the rod was then connected, posterolateral and posterior fusion were performed by using the removed bony fragments and autologous iliac bone grafts. For patients with less severe comminution in the upper endplate of the fractured vertebrae, interbody fusion was performed at the same time.

\section{Research methods}

To assess the effectiveness of decompression, the amount of spinal canal encroachment by bony fragments and the recovery level of neurologic deficits were compared. The amount of the encroachment of bony fragments onto the spinal canal was compared on pre-operative and immediate post-operative CT scans with the average amount of encroachment by bony fragments under an assumption that the average spinal canal cross sectional area of the superior and inferior vertebra in the fractured vertebra as a normal figure. The degree of neurologic deficits was assessed by Frankel grade and the American Spine Injury Association (ASIA) scale, and pre-operative and final follow-up results were compared. The ASIA scale measured the motor power score on the basis of 50 points and sensory score on the basis of 36 points for light touching below L1 dermatome. In addition, to assess the correction of the local kyphotic angles of fracture sites, the angle formed by the upper endplate of superior adjacent vertebra and the lower endplate of fractured vertebra (Fig. 2) was measured pre- and postoperatively. To examine whether kyphotic deformity increased during the follow-up, the local kyphotic angle was also measured and compared at the last follow-up. In addition, the operation hours and estimated blood loss (EBL) were discussed, too.

\section{Results}

The average age of the study subjects was 37 years $( \pm$ 11.7 years), there were 9 male patients (69\%) and 4 female patients $(31 \%)$. The average follow-up period was 30 months ( \pm 13.5 months). Pre-operative diagnoses were made according to McAfee' s method [5]. Ten patients sustained unstable burst fractures and 3 patients sustained translational injuries (fracture-dislocation). Surgery was performed as early as possible, provided the patients were fit for surgery. The average operation time was $168 \mathrm{~min}$ - utes. Steroids were not administered to patients admitted more than 8 hours post-injury. Even within 8 hours of sustaining an injury, steroids were not administered if immediate decompression surgery could be performed. Therefore, a high dose steroid therapy for treating spinal cord injuries was only applied to 2 patients. Intra-operatively, laminar fractures were observed in 10 patients. Among them, 3 patients sustained concurrent dura injuries, for which dural repair was performed. In patients without laminar fractures, no dura injury was observed. The average intraoperative EBL was 2,173 ml. Four patients experienced massive bleeding of more than $3,000 \mathrm{ml}$, and three of them sustained combined injuries, such as extremity fractures or internal organ injuries requiring surgery.

Six patients whose comminution of the upper end plate of

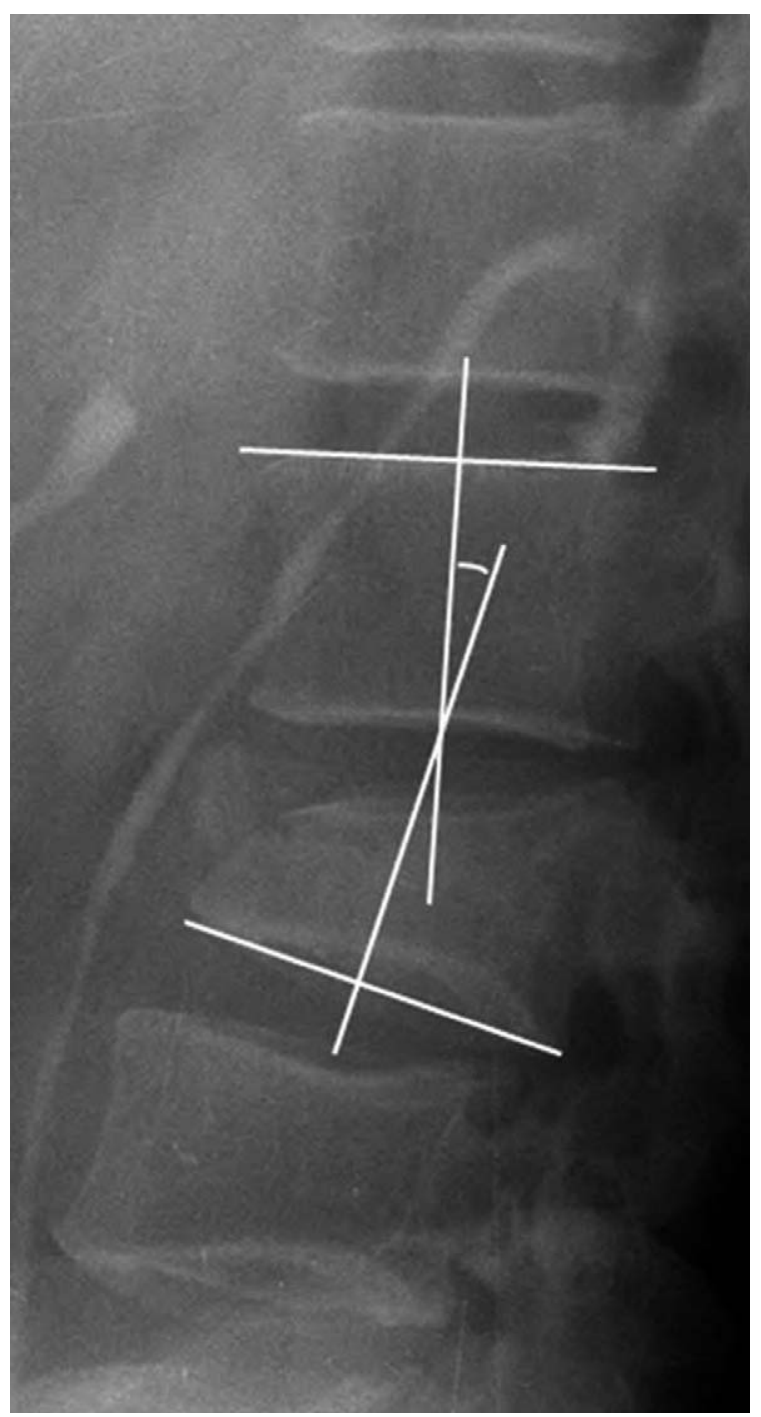

Fig. 2. Measurement technique for segmental kyphosis angle. Measured by Cobb's method at fractured segment. 
the fractured vertebrae were not severe, underwent posterior interbody fusion simultaneously with decompression. During the fusion, fragmented local bones acquired from the decompression procedure or autologous iliac bone from PSIS (posterior superior iliac spine) were inserted alone or together with cages.

Pre-operative canal encroachment was on average $83 \%$ $( \pm 16 \%)$, and post-operative was on average $9 \%( \pm 10 \%)$ $(p<0.01)$. Approximately $74 \%$ of the canal cross sectional area attained recovery through surgery. Regarding the degree of neurologic deficit by Frankel grade, 5 patients were classified as grade A, and 1, 6 and 1 patients were respectively classified as grade $\mathrm{B}, \mathrm{C}$, and D. Concerning post-operative grade, no patient was classified as grade A, and respectively 1, 9 and 3 patients were classified as grade $\mathrm{B}, \mathrm{D}$, and $\mathrm{E}$. Among the 13 study patients, neurological improvement was observed in 12 (92\%). Among patients who showed improvements, an average improvement grade of 1.7 according to Frankel's grading was observed. Specifically, neurological recovery was observed in all patients who suffered complete neurologic deficits. Deterioration of post-operative neurologic deficits was not observed in any patients. The pre-operative motor power score was on average 17 points ( \pm 15 points), while the post-operative was 40 points $( \pm 9$ points $)(p<0.01)$. The sensory score improved from an average of 15 points ( \pm 11 points) to 33 points $( \pm 5$ points) in the post operation $(p<0.01)$ (Table 1).

Concerning change in local kyphotic angle before and after operations, an average of $20.5^{\circ}\left( \pm 10^{\circ}\right)$ kyphotic deformity was observed pre-operatively. The immediate post-operative kyphotic angle improved to approximately $1.5^{\circ}\left( \pm 4.7^{\circ}\right.$ ). And at the final follow-up, it was 3.0 ( \pm $5.5^{\circ}$ ) on average. As such, loss of reduction of kyphotic deformity was observed. At the final follow-up, except for one patient, movements on lateral flexion-extension radiography could not be observed, and on anteroposterior views, solid bone union could be confirmed. In one patient who was in the 40th month after the operation, non-union and instrument failure was observed. Solid union could be achieved after performing anterior and posterior fusion.

\section{Case 1}

A 16-year-old female patient sustained an unstable burst fracture of L2 after a fall. With a complete spinal cord injury demonstrated on admission, her motor power score

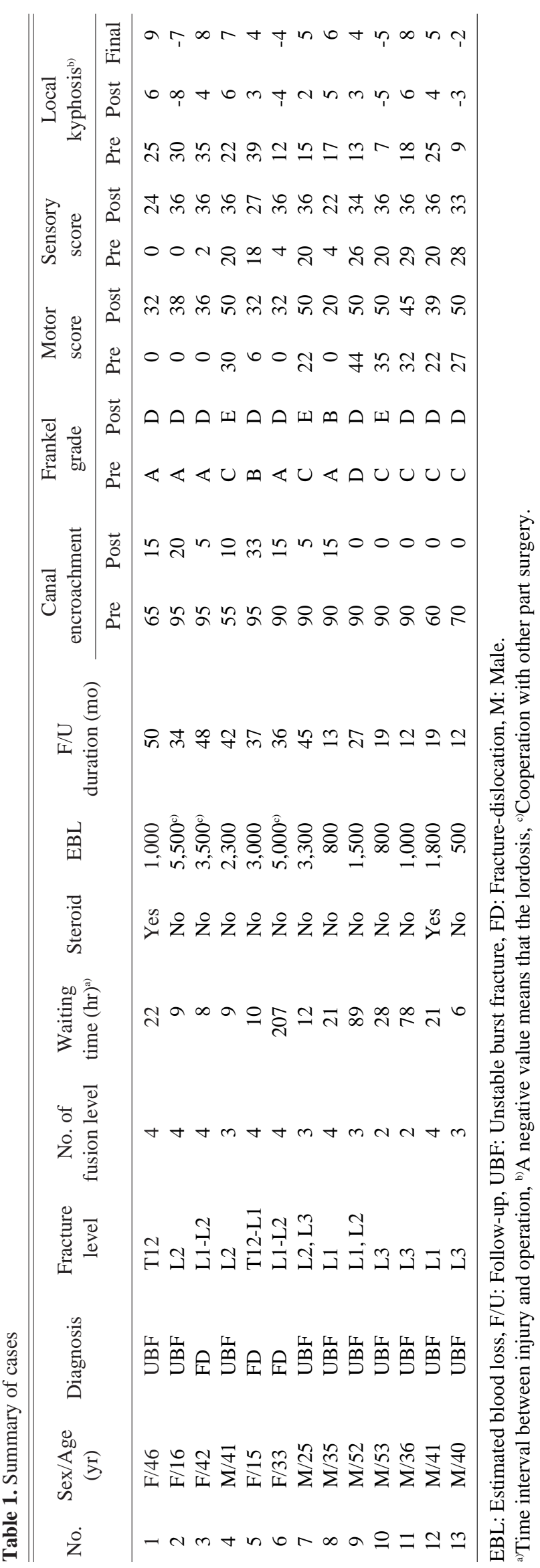


of the lower extremity was 0 point, the sensory score was 0 point, and Frankel grade was A. Pre-operative canal encroachment of the L2 bony fragment was $95 \%$. The bony fragments were removed by left total pediculectomy. Intraoperatively, approximately $5,500 \mathrm{ml}$ of bleeding occurred, but the patient maintained normal vital signs throughout. Post-operative canal encroachment was $20 \%$, and some bony fragments in the right side were not removed. One month after surgery, her lower motor power score was 4 points, while the sensory score was 4 points. Fifteen months after surgery, her motor power score improved to 28 points. Forty-eight months after surgery, at the final follow-up, her motor power score of lower extremity was 38 points, sensory score was 36 points, and Frankel grade D neurological
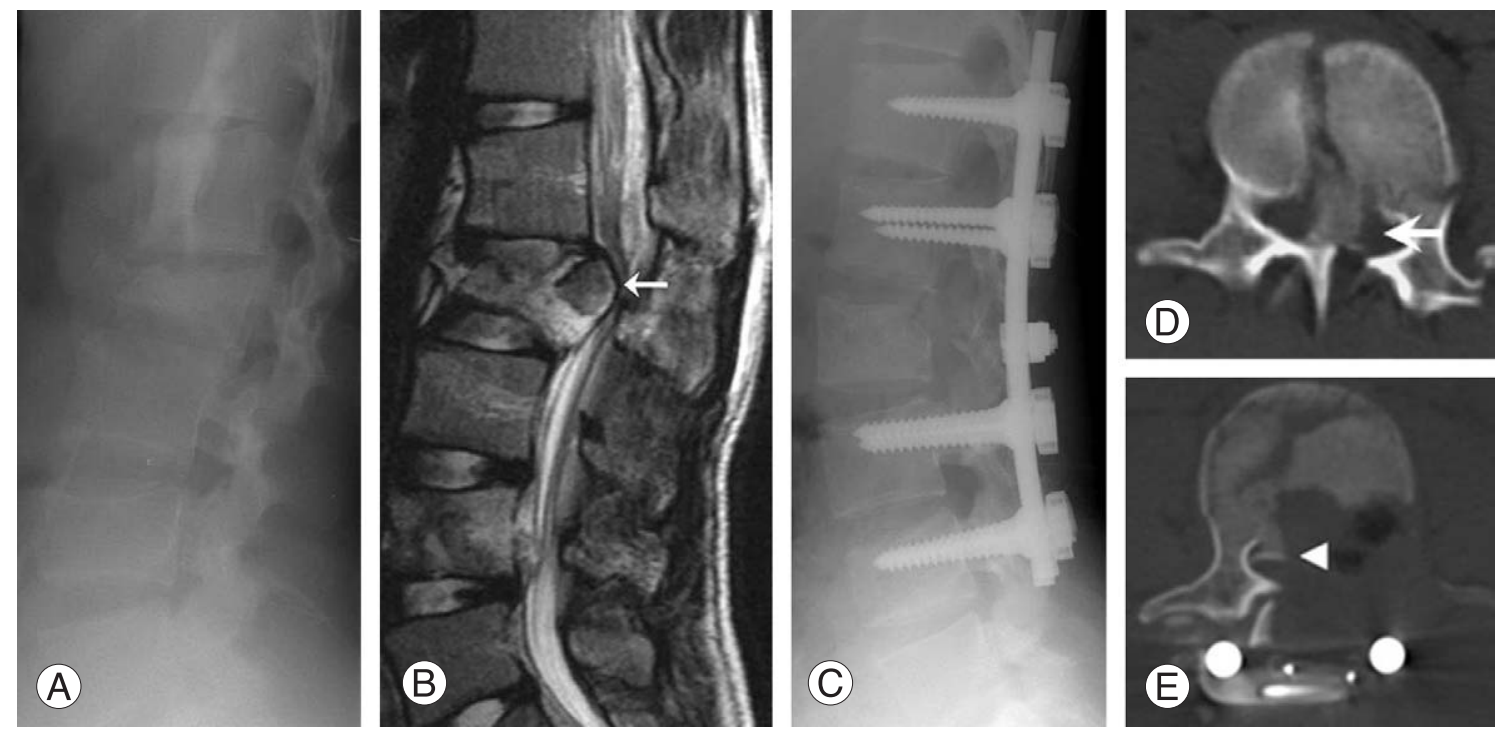

Fig. 3. Images of L2 unstable burst fracture in an 18-year-old girl. Preoperative X-ray (A), magnetic resonace imaging (B) and computed tomography-scan (D) show L2 burst fracture with retropulsed bony fragment (white arrow). After decompression via left pedicle removal and fusion (C, E), some fragment remain at right side (arrowhead). But canal encroachment is much improved and main fragments are removed.
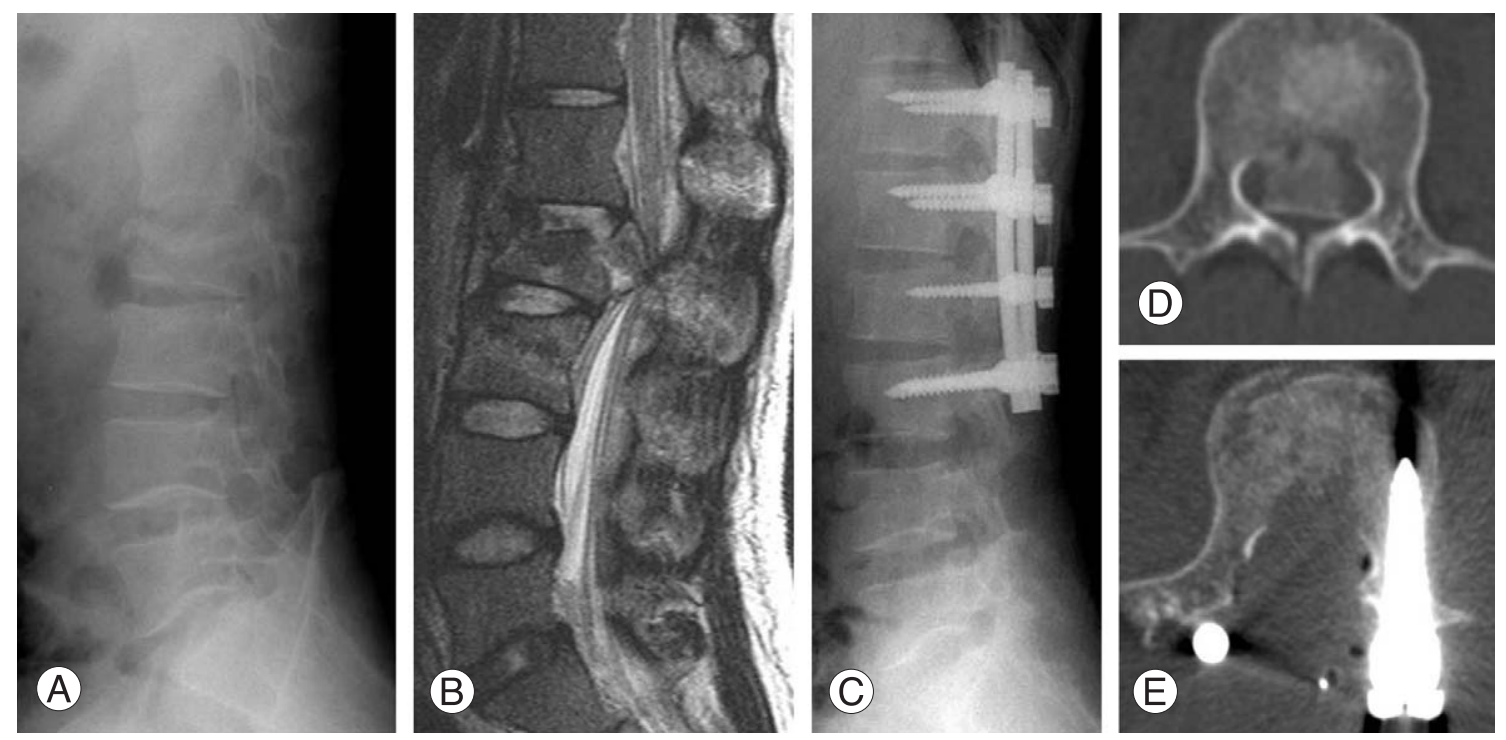

Fig. 4. Images of L2 unstable burst fracture in a 25-year-old man. Preoperative X-ray (A) and magnetic resonance imaging (B) show L2 burst fracture and L3 compression fracture. Spinal canal encroachment by bony fragments is almost $90 \%$ (D). The fragments are removed and canal is restored after decompression (E). Local kyphosis is improved (C). 
recovery was observed (Fig. 3).

\section{Case 2}

A 25-year-old male sustained an unstable burst fracture of L2 and a stable burst fracture of L3 after a fall, and sustained a $90 \%$ canal encroachment by the L2 bony fragment. The pre-operative motor power score and sensory score were 22 points and 20 points, respectively, and Frankel grade $\mathrm{C}$ neurologic deficit was observed. L2 total laminectomy was performed. Based on the sagittal images of preoperative CT, the amount of laminectomy for the superior adjacent vertebra and the sagittal length of retropulsed bony fragments were expected, prior to performing surgery (Fig. 1). Bony fragments were removed by $L 2$ right partial pediclulectomy, and all bony fragments encroached onto the spinal canal were removed. Three months post-operation, his motor power score was 46 points, and sensory score improved to 36 points. Eighteen months post-operation, his motor power score improved to 50 points (Fig. 4).

\section{Discussion}

The lower thoracic and lumbar spine is biomechanically most susceptible to injuries, so spine injuries occur most frequently in these areas $[4,6]$. It remains controversial whether surgical treatments are required in patients who sustain lower thoracic and lumbar fractures. It has been reported that conservative management is adequate for patients who sustain stable fractures without associated neurologic deficits [7]. On the other hand, some authors assert that surgical intervention is required to reduce risks of delayed neurologic deficits or kyphotic deformity triggering the pain [8]. Generally, surgical intervention is considered mandatory for patients who sustain fractures with associated neurologic deficits.

It is thought that in burst fractures, the more severe canal encroachment by bony fragments, the more severe the degree of neurologic deficit. Nonetheless, canal encroachment by bony fragments does not directly indicate the degree of neurologic deficit; the degree of neurologic deficit is thought to be associated with the severity of impact at the time of injury. In a cadaver study, Panjabi et al. [9] reported that dynamic canal encroachment at the time of fracture is approximately $85 \%$ larger than the static state, and thus it is difficult to predict the actual level of neurologic deficit by canal encroachment detected by plain radiography or CT.
However, generally, more than $50 \%$ of canal encroachment is an indication for surgical intervention [10]. It has been shown that continuous mechanical compression of the neural structure hinders neurological recovery, due to edema and circulation disorder. Thus, decompression is said to more helpful to neurological recovery [11].

There are two decompression methods: indirect and direct decompression. Indirect decompression is a method to reduce displaced bony fragments by ligamentotaxis within 48-72 hours after injury. This method reduces bony fragments by adding tension to the posterior longitudinal ligament (PLL) or posterior annulus. However, if the canal encroachment is more than $50 \%$ or the PLL is considered to have lost continuity because of rotation observed in bony fragments on radiography, this method is not effective and will probably need direct decompression. In all patients engaged in the study, the pre-operative canal encroachment by bony fragments showed $50 \%$, thus necessitating direct decompression.

There are three surgical approaches to direct decompression: the anterior approach, posterior approach and the combined approach. The optimal method remains controversial. The anterior approach has generally been applied to patients who sustained severe neurologic deficits or kyphotic deformity [12]. This approach has advantages in that it enables removal of bony fragments causing neurologic deficits under clear view, and correction of kyphotic deformity. Other advantages include high bone union rates and less correction loss due to rigid fixation [13,14]. However, the anterior approach is technically demanding and requires long operation time. The incidence of complications, such as bleeding, vascular injury, pneumothorax, post-operative pneumonia is also high. In addition, it is difficult to perform the approach in patients compromised by reduced pulmonary function, severe thoracic injury and those with concurrent abdominal injuries.

The posterior approach is a method familiar to most spinal surgeons. The approach ensures safe exposure of the operation field and imposes low risk of injuring internal organs, including the lungs and vessels. In addition, it is effective for correction of the vertebral alignment. If pedicle screws are used, fixation spanning 3 columns of vertebrae is feasible, thus rigid fixation can be achieved. In addition, it enables gross inspection of neural tissues in patients undergoing laminectomy. Regarding decompression effectiveness, it has been reported that adequate results could be obtained by using the posterior approach. Danisa et al. [15] 
reported similar levels of neurological recovery in 49 thoracolumbar burst fracture patients treated with all three spinal decompression methods described above. In addition, adequate spinal canal decompression and satisfactory neurological recovery could be achieved by anterior impaction of bony fragments via the posterior approach $[3,16]$. However, it is difficult to accurately assess the extent of bony reduction in the operating field when anterior impaction of posteriorly displaced bony fragments was performed due to massive bleeding. If fracture sites re-collapse post-operatively, posterior displacement of the impacted bony fragments may occur again in some patients, which could result in delayed neurologic deficit $[17,18]$. Therefore, the authors did not perform impaction of large bony fragments compressing the nerves, but opted to remove them to achieve adequate decompression. Canal encroachment, which stood at $83 \%$ pre-operatively, fell to 74\% owing to this approach. Residual canal encroachment was on average $9 \%$ and so adequate decompression could be achieved. Regarding the degree of neurological recovery, satisfactory outcomes could also be achieved.

Short-level posterior fusion using pedicle screws was introduced by Dick et al. [19] as a concept dubbed 'fixateur interne.' It has subsequently been used as a mainstay of surgical treatment for burst fractures. However, when performing short-level posterior fusion, gaps are generated within the fractured vertebra in accordance with fracture reduction, thus reducing load transmission via anterior column. Because lack of anterior support, it has been reported that in patients who underwent short-level fusion, kyphotic deformity recurs frequently, and the higher rate of instrumental failure is likely [20,21]. To prevent such complications, some authors assert that it is important to reinstate anterior support by impaction of bony fragment [3], or by filling bone cement through kyphoplasty [22]. On the other hand, Tezeren and Kuru [23] reported that in patients who underwent long-level posterior instrumentation and fusion, good results could be achieved without reinstating anterior support. In this study, we couldn't expect anterior support that can be achieved by impaction of bony fragments, because we removed posteriorly displaced bony fragments. This raised the risks of relapse of kyphotic deformity and breakage of instrumentation. Therefore, the authors performed long segment fusion to reduce the complications, when such risks were considered high due to removals of large bony fragments. In addition, for the fractured vertebra, we tried to prevent against excessive loading to the rod at the fracture sites by inserting pedicle screws in the case of no comminution in the pedicle on the opposite side of the approach side. Lee et al. [24] reported that insertion of pedicle screws into the fractured vertebra is a safe and effective method for the treatment of unstable thoracolumbar burst fractures. As seen in the results, the post-operative loss of correction was approximately $1.5^{\circ}$, and correction of kyphotic deformity was well maintained. Except for one patient, complications such as instrumental failure did not occur. However, the follow-up observation period was relatively short, and the number of patients included in the study was small. Studies encompassing larger number of patients with a longer period of follow-ups are required to validate our findings.

According to previous reports, patients who sustained complete paraplegia above the upper- or mid-thoracic level experience difficulties with neurologic recovery. However, in patients who experience complete paraplegia below the lower thoracic level, neurological recovery has been observed. In a study conducted on 40 complete paraplegic patients with lower thoracic or lumbar fracture, Prabhakar et al. [4] reported that improvement by more than 1 grade of ASIA impairment scale had been observed in almost $90 \%$ of the patients. In patients who underwent aggressive decompression, the degree of neurological recovery was better than those who underwent posterior fusion alone. In our study, similarly, in all 5 patients who experienced complete paraplegia below the injury level, neurological recovery was observed. It is thought to be due to vertebra and spinal cord anatomy. The major nerve root to the lower extremity originates from lumbar enlargement of the spinal cord existing between $\mathrm{T} 9$ and $\mathrm{T} 12$. In the area between $\mathrm{T} 12$ and L1, the conus medullaris is present, and the spinal cord terminates at the L1-2 disc level. Therefore, only nerve roots are present below the L1-2 disc level. As such, recovery of motor and sensory function of the lower extremity in complete spinal cord injury above ninth thoracic vertebra (T9) is almost impossible, because it is an injury to the central nerve system. On the other hand, neural injury developed in the distal part of L1-2 disc level constitutes injury to the peripheral nerve system. Thus, it is thought that even when evidence of complete paraplegia is seen immediately after injury, there may be a possibility of recovery. In this study, despite evidence of complete paraplegia below the level of injury due to fracture-dislocation of the second lumbar vertebra, neurological recovery was seen following surgery. In addition, an injury at the level of T11 or T12, 
constitutes a combined injury of the peripheral nerve of the upper lumbar nerve root (L1, 2, and L3 nerve root) and the central nerve (anterior horn cell of L4, 5, and S1 root and the pyramidal tract). As such, recovery of the peripheral nerve of the upper lumbar nerve root may be anticipated.

The limitations of this study are as follow: it is a retrospective study encompassing a small patient population. In addition, surgical outcomes were not compared with that of the anterior approach. As such, further studies are required to validate our findings. In addition, it should be kept in mind that during removal of bony fragments, massive bleeding occurs, and consequently, the possibility of complications should be kept in mind. The authors assert that the ideal indication for the posterior approach is fractures at the distal to lower thoracic level associated with the following: from moderate to severe neurologic deficits (motor grade lower than grade 3), bony fragments compressing the nerve are clear, and the patients with favorable general conditions. It is thought that better results could be obtained by conducting intraoperative nerve monitoring or taking intraoperative CT.

\section{Conclusions}

The posterior approach can be considered one of methods to treat lower thoracic and lumbar fractures. In patients with lower thoracic and lumbar fractures combined with complete or incomplete neurologic deficits, this approach can remove bony fragments compressing the neural tissues and perform posterior fusion using pedicel screws. It helps achieve sufficient spinal canal decompression and neurological recovery without post-operation instability or increases in kyphotic deformity, and also helps attain desirable clinical results.

\section{REFERENCES}

1. Shi R, Liu H, Zhao X, et al. Anterior single segmental decompression and fixation for Denis B type thoracolumbar burst fracture with neurological deficiency: thirty-four cases with average twenty-six month follow-up. Spine (Phila Pa 1976) 2011;36:E598-605.

2. Hitchon PW, Torner J, Eichholz KM, Beeler SN. Comparison of anterolateral and posterior approaches in the management of thoracolumbar burst fractures. J Neurosurg Spine 2006;5:117-25.

3. Kong W, Sun Y, Hu J, Xu J. Modified posterior decom- pression for the management of thoracolumbar burst fractures with canal encroachment. J Spinal Disord Tech 2010;23:302-9.

4. Prabhakar MM, Rao BS, Patel L. Thoracolumbar burst fracture with complete paraplegia: rationale for secondstage anterior decompression and fusion regarding functional outcome. J Orthop Traumatol 2009;10:83-90.

5. McAfee PC, Yuan HA, Fredrickson BE, Lubicky JP. The value of computed tomography in thoracolumbar fractures: an analysis of one hundred consecutive cases and a new classification. J Bone Joint Surg Am 1983;65:461-73.

6. Dai LY, Jiang SD, Wang XY, Jiang LS. A review of the management of thoracolumbar burst fractures. Surg Neurol 2007;67:221-31.

7. Mumford J, Weinstein JN, Spratt KF, Goel VK. Thoracolumbar burst fractures: the clinical efficacy and outcome of nonoperative management. Spine (Phila Pa 1976) 1993;18:955-70.

8. Denis F, Armstrong GW, Searls K, Matta L. Acute thoracolumbar burst fractures in the absence of neurologic deficit: a comparison between operative and nonoperative treatment. Clin Orthop Relat Res 1984;(189):142-9.

9. Panjabi MM, Kifune M, Wen L, et al. Dynamic canal encroachment during thoracolumbar burst fractures. J Spinal Disord 1995;8:39-48.

10. Willén J, Anderson J, Toomoka K, Singer K. The natural history of burst fractures at the thoracolumbar junction. J Spinal Disord 1990;3:39-46.

11. Bradford DS, McBride GG. Surgical management of thoracolumbar spine fractures with incomplete neurologic deficits. Clin Orthop Relat Res 1987;(218):201-16.

12. Verlaan JJ, Diekerhof CH, Buskens E, et al. Surgical treatment of traumatic fractures of the thoracic and lumbar spine: a systematic review of the literature on techniques, complications, and outcome. Spine (Phila Pa 1976) 2004;29:803-14.

13. Song KJ, Lee KR, Jeon JY. Surgical treatment for the thoracolumbar burst fractures with neurologic deficit: a comparative study between anterior and posterior approach. J Korean Orthop Assoc 2000;35:154-60.

14. Haas N, Blauth M, Tscherne H. Anterior plating in thoracolumbar spine injuries: indication, technique, and results. Spine (Phila Pa 1976) 1991;16(3 Suppl):S100-11.

15. Danisa OA, Shaffrey CI, Jane JA, et al. Surgical approaches for the correction of unstable thoracolumbar burst fractures: a retrospective analysis of treatment outcomes. J Neurosurg 1995;83:977-83. 
16. Cho KJ, Moon KH, Kim MG, Ko SM, Park HW, Park SR.

Posterolateral decompression and posterior instrumentation in lumbar and thoracolumbar burst fracture with neurologic deficit. J Korean Soc Spine Surg 2000;7:234-9.

17. Vornanen MJ, Böstman OM, Myllynen PJ. Reduction of bone retropulsed into the spinal canal in thoracolumbar vertebral body compression burst fractures: a prospective randomized comparative study between Harrington rods and two transpedicular devices. Spine (Phila Pa 1976) 1995;20: 1699-703.

18. Been HD, Bouma GJ. Comparison of two types of surgery for thoraco-lumbar burst fractures: combined anterior and posterior stabilisation vs. posterior instrumentation only. Acta Neurochir (Wien) 1999;141:349-57.

19. Dick W, Kluger P, Magerl F, Woersdörfer O, Zäch G. A new device for internal fixation of thoracolumbar and lumbar spine fractures: the 'fixateur interne'. Paraplegia 1985; 23:225-32.

20. Sasso RC, Renkens K, Hanson D, Reilly T, McGuire RA Jr, Best NM. Unstable thoracolumbar burst fractures: ante- rior-only versus short-segment posterior fixation. J Spinal Disord Tech 2006;19:242-8.

21. McLain RF, Sparling E, Benson DR. Early failure of shortsegment pedicle instrumentation for thoracolumbar fractures: a preliminary report. J Bone Joint Surg Am 1993; 75:162-7.

22. Marco RA, Meyer BC, Kushwaha VP. Thoracolumbar burst fractures treated with posterior decompression and pedicle screw instrumentation supplemented with balloonassisted vertebroplasty and calcium phosphate reconstruction. Surgical technique. J Bone Joint Surg Am 2010;92 Suppl 1 Pt 1:67-76.

23. Tezeren G, Kuru I. Posterior fixation of thoracolumbar burst fracture: short-segment pedicle fixation versus longsegment instrumentation. J Spinal Disord Tech 2005;18: 485-8.

24. Lee JH, Jun DS, Shin WJ, Ahn SJ. The effect of pedicle screw instrumentation on fractured vertebrae in unstable thoracolumbar burst fractures with canal encroachment and clinical result. J Korean Soc Spine Surg 2006;13:10-5. 